\title{
Successful Decannulation of Hybrid ECMO Circuitry: The First Philippine Experience
}

Renato C. Ong, Jr., RMT, M.D., FPCP, ISMPP ${ }^{1,2}$; Maria Stephanie Alessa R. Sales-Florentino, MD, FPCP ${ }^{1,2}$; Frederick H. Verano, MD, FPCP, FPSN ${ }^{1,3}$; Rod T. Castro, MD, FPCP, FPCC, FPCCM 1 , 2; Jillian Mae L. Tabora-Lacdao, MD, FPCP, FPCCP', 4; Raul L. Lapitan, MD, FPCP, FPCC, FACC ${ }^{1,2}$

${ }^{1}$ Department of Medicine

2 Section of Cardiology

${ }^{3}$ Section of Nephrology

${ }^{4}$ Section of Pulmonology, Makati Medical Center, Makati City, Philippines.

Main Author: Renato C. Ong, Jr., RMT, M.D., FPCP, ISMPP

Orcid ID No: 0000-0002-0433-7511

E mail: r.ongjrmd@gmail.com

Address for correspondence: Renato C. Ong Jr., MD. Section of Cardiology Makati Medical Center 6FT2 2 Amorsolo Street, Legaspi Village, Brgy. San Lorenzo, Makati City, Philippines 1229. Mobile No. +639171859797. Telephone No. +6388888999.

\begin{abstract}
We report the first VA-VAV-VV ECMO conversion in a 57-year-old Filipino female with persistent coronary insufficiency from toxic shock syndrome due to Streptococcus pyogenes bacteremia, acute respiratory distress syndrome, and Harlequin syndrome with progressive acute limb ischemia from cone snail venom poisoning.
\end{abstract}

The patient came in via air ambulance transport because deteriorating clinical status after having stepped on a cone snail 3 days prior and developing severe dehydration from vomiting and passage of voluminous watery stools after eating a local delicacy. The patient was admitted at the ICU where after 3 days of treatment, the patient developed cardiac tamponade and underwent stat pleuropericardial windowing with pericardiocentesis.

Due to increasing pressor requirements from the combined shock, Swan-Ganz catheterization was inserted and a VA-ECMO system was set up. There was gradual improvement in cardiac hemodynamics, however oxygen requirement was increasing and both lower extremities became progressively violaceous with decreasing pulses. Harlequin syndrome was ruled in, the multidisciplinary team decided to convert to VAV hybrid circuit by adding a venous access by way of the right internal jugular vein. Improvement in oxygenation lead to eventual conversion from VAV to purely VV dedicated circuit. Ultrafiltration via ECMO was likewise done because of worsening azotemia and oliguria.

The patient was then gradually weaned off from ECMO and was successfully decannulated after 8 days. Below knee amputation was done to address the progressive acute limb ischemia. The patient was nutritionally built up and physically rehabilitated and was eventually discharged improved on the 28th hospital day.

\section{CASE REPORT}

A 57-year old female with unremarkable past medical history presented in our institution due to lose stools and vomiting. History revealed that 3 days prior to consult, she developed $\mathrm{R}$ distal lower extremity swelling and tenderness after accidentally stepping on a cone snail while snorkeling in Siargao. She had 3 episodes of voluminous, watery stools and vomiting after eating "talakitok". Initially seen at a local hospital hypotensive, IV boluses of crystalloids were given, and Norepinephrine drip was started, and then she was transferred via air ambulance to our institution.

Upon arrival at the ED, she was hypotensive with a MAP of $50 \mathrm{mmHg}$, tachycardic at $110 \mathrm{bpm}$, tachypneic at $28 \mathrm{cpm}$ and febrile at $40.1^{\circ} \mathrm{C}$. She was able to talk in words to a few interrupted phrases, had flushed, dry skin with parched buccal mucosae and flat neck veins. There was a poorly healing puncture wound with swollen, patchy erythemato-violaceous discoloration and tenderness along on the $\mathrm{R}$ plantar area.

Initial isolate from blood culture was Streptococcus pyogenes and culture-guided antimicrobials were started. Inflammatory markers and cardiac biomarkers were all significantly elevated. Echocardiogram showed a small pericardial effusion with RV diastolic collapse, dilated LV, eccentric LV hypertrophy with global hypokinesia, depressed EF of $35 / 31 \%$ and grade I LV diastolic dysfunction with normal LV filling pressures. Dobutamine and Dopamine were started, and she underwent stat pleuropericardial windowing with pericardiocentesis draining 200-ml non-clotting serosanguinous pericardial and 400-ml pleural fluid.

With increasing vasopressor requirements and persistent clinical deterioration, the patient underwent VA-ECMO cannulation via a $21 \mathrm{~F}$ drainage cannula from the $L$ femoral vein and a $19 \mathrm{~F}$ infusion cannula into the $L$ femoral artery with Swan-Ganz catheterization. Along the course, vasopressor requirements decreased, and hemodynamic status improved, however the oxygen requirement increased. Serial chest radiographs showed worsening of the hazy opacities to full blown acute respiratory distress syndrome (Figure 1). She developed Harlequin syndrome and limb ischemia, the multidisciplinary team converted the VA to a VAV hybrid circuit by way of $21 \mathrm{~F}$ drainage cannula into the $R$ internal jugular vein (Figure 2 and 3). Subsequent improvement in oxygenation was observed (Figure 4) and the hybrid circuit was thenceforth converted to a purely $\mathrm{V} V$ dedicated track by clamping the $L$ femoral artery access (Figure 2c). 
Figure 1: Chest radiographs of the patient. A. Normal baseline radiograph. B. Post-VA ECMO, there was accentuation of pulmonary vascular markings with some hazy densities in the left parahilar and paracardiac areas due to pulmonary congestion and edema. $\mathrm{CO}, \mathrm{CI}, \mathrm{SVR}$, and SVRI improved, cardiac biomarkers significantly decreased. PF ratio decreased. C. Day 1 Post-VA ECMO, there was worsening of pulmonary opacities. D. Day 2 Post-VA ECMO development of acute respiratory distress syndrome. CO, Cl, SVR, and SVRI significantly improved, cardiac biomarkers further decreased. Oxygen requirement significantly increased, and PF ratio worsened.

VA ECMO, venoarterial extracorporeal membrane oxygenation. CO, cardiac output. Cl, cardiac index. SVR, systemic vascular resistance. SVRI, systemic vascular resistance index.

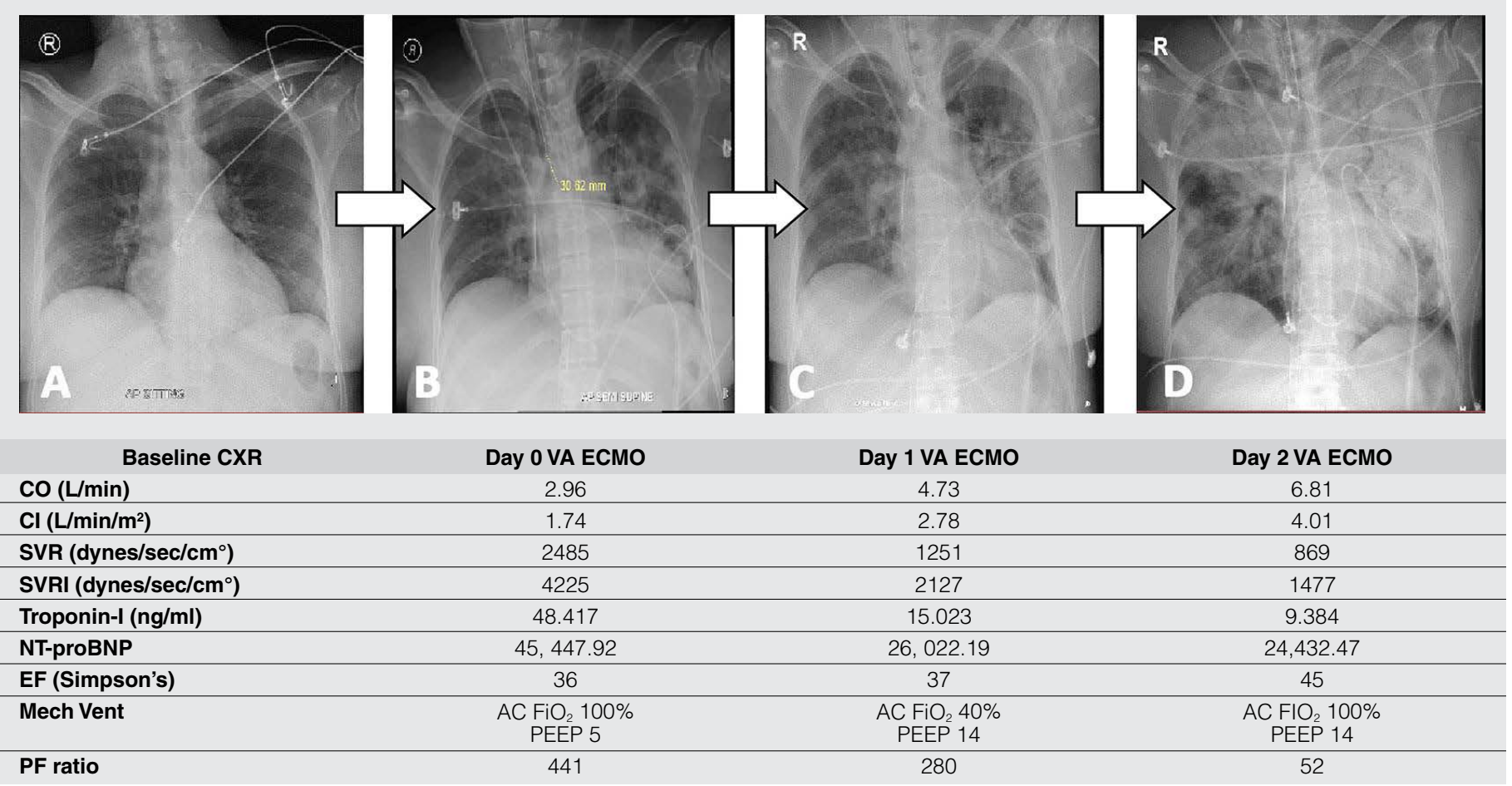

Figure 2: Different ECMO modalities in our patient. A, VV mode - a $21 \mathrm{~F}$ drainage cannula from the left femoral vein and a $19 \mathrm{~F}$ infusion cannula into the left femoral artery; B, VAV mode - a 21F drainage cannula was introduced by way of the $R$ internal jugular vein and connected to the VA circuit; and C, VV mode - the left femoral artery was clamped.
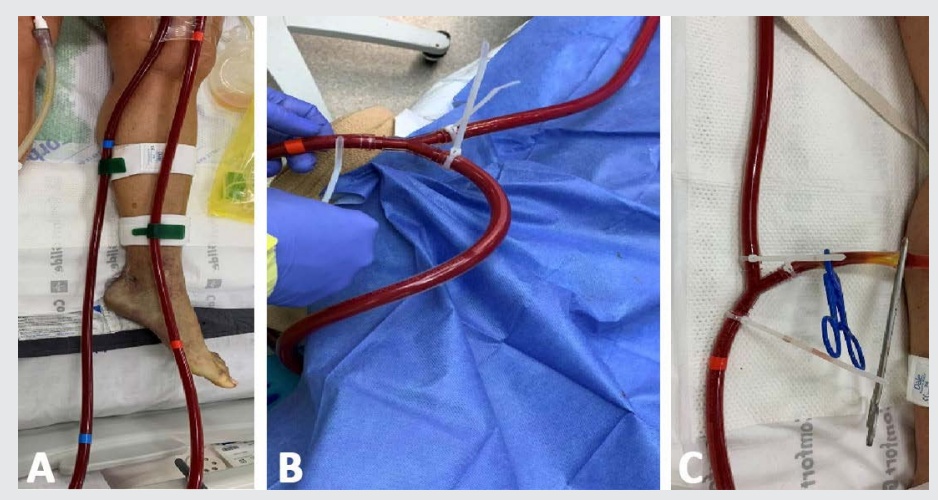

Figure 3: Chest radiographs of the patient in transition to VA-VAV ECMO. A. VA ECMO. ABG showed severe ARDS with a very low $\mathrm{pO}_{2}$. B. Post-VAV, further worsening of pulmonary infiltrates but with significant improvement of $\mathrm{PF}$ ratio and $\mathrm{pO}_{2}$.

VA ECMO, veno-arterial extracorporeal membrane oxygenation, VAV ECMO, veno-arterial-venous extracorporeal membrane oxygenation, ARDS, acute respiratory distress syndrome.

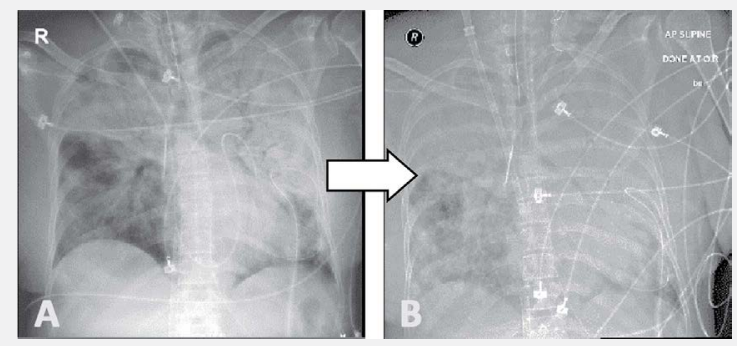

\begin{tabular}{|c|c|c|}
\hline & VA ECMO & VAV ECMO \\
\hline $\mathrm{pO}_{2}$ & 54 & 236 \\
\hline pH & 7.38 & 7.32 \\
\hline $\mathrm{pCO}_{2}$ & 45 & 52 \\
\hline $\mathrm{HCO}_{3}$ & 26.6 & 26.8 \\
\hline $\mathbf{S P O}_{2}$ & 93.5 & 100 \\
\hline PF & 54 & 236 \\
\hline
\end{tabular}


Figure 4: Chest radiographs of the patient in VV ECMO. A. Day 3 VV ECMO with confluent parenchymal infiltrates with blunting of the bilateral costrophrenic sulci with her maintaining acceptable oxygenation. B. Day 5 VV ECMO, significant clearing of bilateral infiltrates with further improvement of $A B G$ parameters and patient is being weaned off from VV ECMO. C. Day 7 VV ECMO, further clearing of the pulmonary infiltrates. ECMO sweep was turned off with physiologic PEEP.

VV ECMO, venovenous extracorporeal membrane oxygenation.

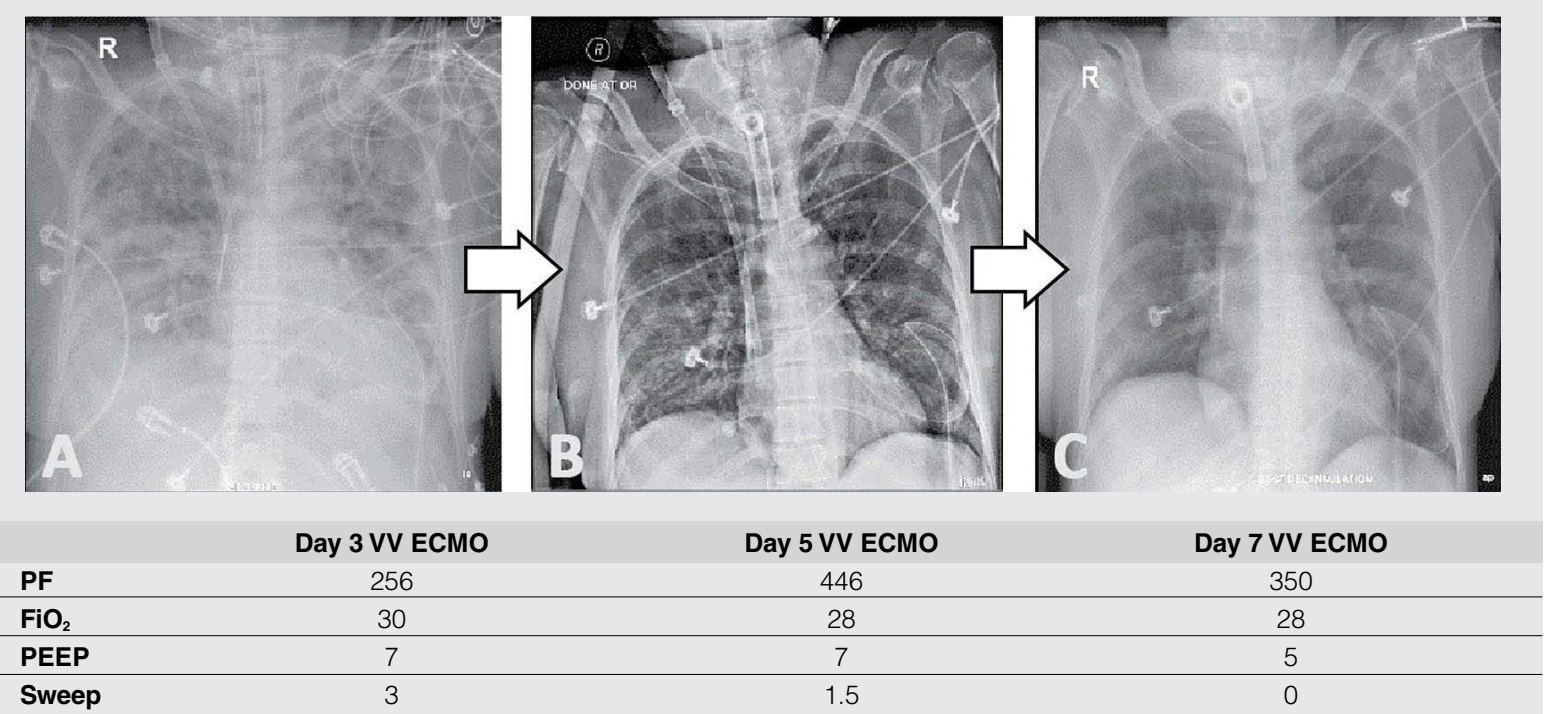

Ultrafiltration via ECMO was initiated when the azotemia worsened and when the patient became oligoanuric. Subsequently, the patient remained hemodynamically stable with gradual restitution of oxygenation and kidney function and was weaned off from both ECMO and ultrafiltration. After 8 days, the patient was decannulated with simultaneous $L$ below knee and $\mathrm{R}$ transmetatarsal amputation (Figure 5) as the pulses became dismal to thump at best by doppler ultrasound. The patient underwent physical reconditioning with nutritional build up. Artificial limb was fitted, and the patient was ultimately discharged significantly improved.

\section{DISCUSSION}

In our patient, blood cultures isolated Streptococcus pyogenes and procalcitonin was 271 times elevated, troponin I was 3,227 times elevated, CK-MB isoenzyme was 15 times elevated, and NT-proBNP was 363 times elevated. Chest X-ray showed accentuation of pulmonary vascular markings with some hazy densities in the left perihilar and paracardiac areas due to pulmonary congestion and edema with $\mathrm{PaO}_{2} / \mathrm{FiO}_{2}$ ratio of 207. Acute myocarditis from toxic shock syndrome with ARDS secondary to Streptococcus pyogenes bacteremia.
Figure 5: Lower extremities of patient during admission. A. edematous right foot, with cyanotic digits. B. Post transmetatarsal amputation with a clean and wellcoaptated stump. C. Cyanotic plantar area of the left foot with areas of pallor in the dorsomedial side. D. Post-below knee amputation of the left lower extremity showing a well coaptated stump with drains in place.
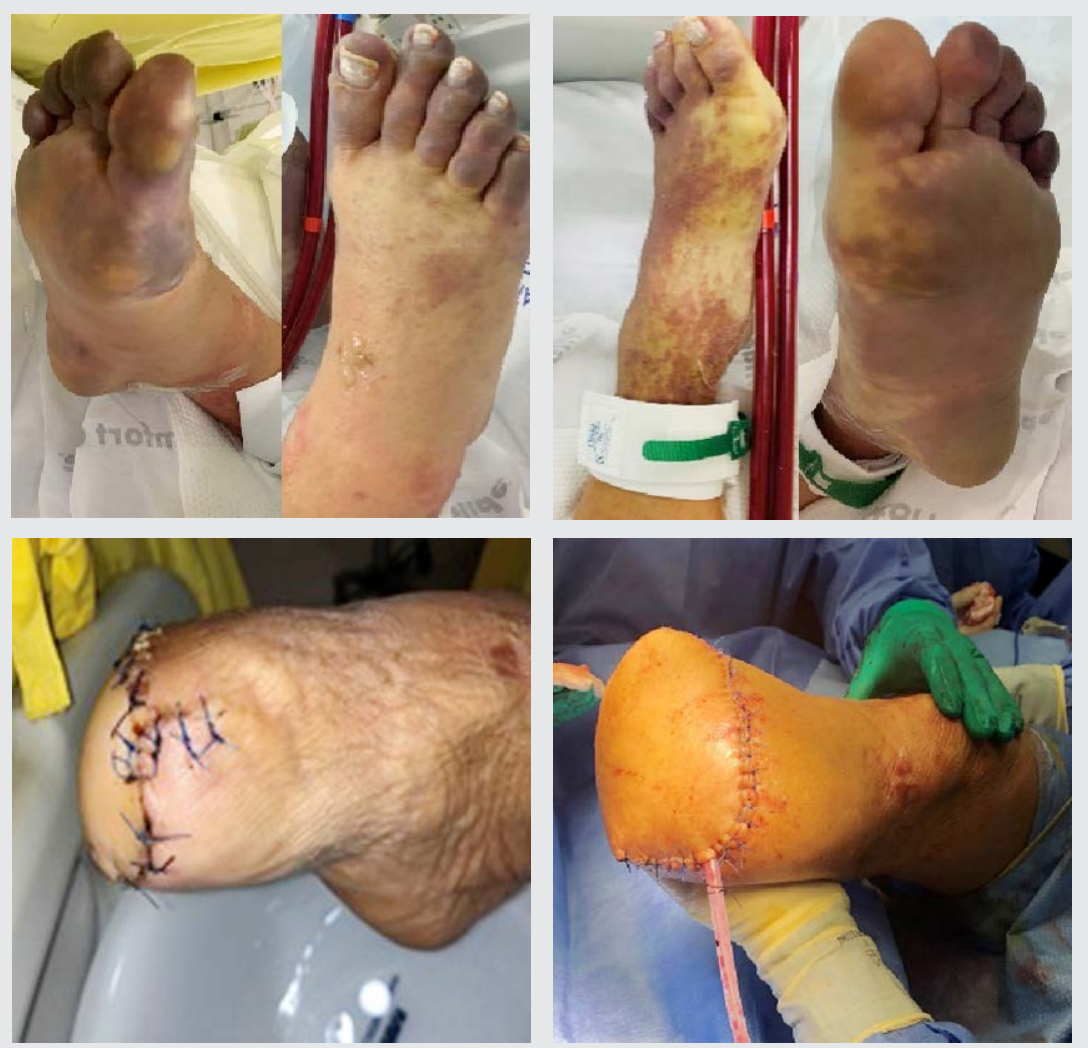
The patient underwent VA ECMO (SAVE Score of -7) with the drain cannula at the $L$ femoral vein and a return cannula at the $L$ femoral artery. A Swan-Ganz catheter was also inserted. On day 1 post VA ECMO, the cardiac output of the patient normalized, vasopressors were titrated down, and the cardiac biomarkers were improving. A 2Decho was done and showed improvement of wall motion contractility of the inferior, inferolateral, lateral wall segments and an increase in EF from 37\% to $45 \%$. Serial chest radiographs showed worsening of pulmonary opacities as well. And her oxygenation status deteriorated, with a PF ratio of only 52.

ECMO is an extreme life-saving procedure providing prolonged but temporary support of heart and/or lung function lasting from days to a few months. Dual cannulation is the most common form for ECMO configuration, i.e., veno-venous (VV) or veno-arterial (VA) cannulation. On the other hand, triple cannulation ECMO is a novel and multifaceted form of mechanical assist where in most cases it is introduced by adjoining a third cannula to and existing the VV- or VA-ECMO circuit. The veno-aterio-venous (VAV) cannulation is used in patients with coexisting severe lung and heart failure, where the drainage cannula draws blood from the right atrium, the ECMO outflow is divided into 2 parts: one toward the aorta and another toward the right atrium, where it represents a mixture of both VV- and VA-ECMO and provides hemodynamic and respiratory support at the same time. This was the strategy employed in our patient.

With extreme cardiac dysfunction on ECMO support, if the heart is not beating and the aortic valve is not opening, the blood being pumped through this cannula provides $100 \%$ of the patient's blood flow. In this scenario there is no mixing of the two circulations since no circulatory support is being provided by the native heart. Upon return of cardiac function, when the heart starts to beat and the aortic valve begins to open, the situation becomes quite unique. And because the peripherally placed ECMO cannula is pumping blood in a direction that is retrograde to the native blood flow, the two circulations merge or mix in a location somewhere between the aortic valve and the arterial cannula. As LV function is restored and contractility increases, the mixing cloud may shift distally in the aorta towards the arterial cannula. The same shift can occur if the ECMO blood flow is decreased. Conversely, as ECMO blood flow is increased or if LV function decreases, the mixing cloud may shift more proximally in the aorta.

To address the differential cyanosis from Harlequin syndrome and worsening hypoxemia from the severe ARDS, conversion of the dual circuit to a triple circuit by adding a $21 \mathrm{~F}$ drainage cannula into the $R$ internal jugular vein and connecting it to the VA circuit. After the oxygenation improved, a pure VV ECMO circuit was eventually maintained where further improvement was noted, paving the way to successful weaning and decannulation.

Keywords: ECMO; VA-ECMO; VV-ECMO; VAV-ECMO; Hyrbid ECMO; Myocarditis; Streptococcus pyogenes; ARDS; Cone snail venom poisoning.

\section{REFERENCES}

1. Abrams D, Combes A, Brodie D. Extracorporeal membrane oxygenation in cardiopulmonary disease in adults. J Am Coll Cardiol. 2014;63:2769-78.

2. Asaumi Y, Yasuda S, Morii I, Kakuchi H, Otsuka Y, Kawamura A, Sasako Y, Nakatani T, Nonogi $\mathrm{H}$, Miyazaki S. Favourable clinical outcome in patients with cardiogenic shock due to fulminant myocarditis supported by percutaneous extracorporeal membrane oxygenation. Eur Heart $\mathrm{J}$. 2005:26:2185-2192. doi: 10.1093/eurhearti/ehi411.

3. Guglin, M. et al. (2019) Venoarterial ECMO for adults. J Am Coll Cardiol. 2019; 73 (6): 698-716.

4. Guenther S, Theiss HD, Fischer M, Sattler S, Peters S, Born F, Pichlmaier M, Massberg S, Hagl C, Khaladj N. Percutaneous extracorporeal life support for patients in therapy refractory cardiogenic shock: initial results of an interdisciplinary team. Interact Cardiovasc Thorac Surg 2014;18:283-291. doi: 10.1093/icvts/ivt505.

5. Lorusso R, Centofanti P, Gelsomino S, Barili F, Di Mauro M, Orlando P, et al. Venoarterial extracorporeal membrane oxygenation for acute fulminan myocarditis in adult patients: a 5-year multi-institutional experience. Ann Thorac Surg. 2016;101(3):919-26.

6. Napp LC, Bauersachs J. Triple Cannulation ECMO, Extracorporeal Membrane Oxygenation - Advances in Therapy, Michael S. Firstenberg, IntechOpen, 2016. DOI: 10.5772/63392.

7. Napp LC, Kuhn C, Hoeper MM, Vogel-Claussen J, Scahfer A, Bauersachs J. Cannulation strategies for percutaneous extracorporeal membrane oxygenation in adults. Clin Res Cardiol. 2016;105:283-296. doi: 10.1007/ s00392-015-0941-1

8. Patel, A. R., Patel, A. R., Singh, S., Singh, S., \& Khawaja, I. (2019). Applied Uses of Extracorporeal Membrane Oxygenation Therapy. Cureus, 11(7), e5163. https://doi.org/10.7759/cureus.5163

9. Quang TN, Dang TN, Van BL, Ngoc QN, Thai BL, et al. (2019) A Patient with ARDS and Cardiogenic Shock Caused by Streptococcus Pyogenes Successfully Treated by V-A-V ECMO. J Surg Open Access. 5(3): dx.doi. org/10.16966/2470-0991.184.

10. Rousse N, Juthier F, Pincon C, Hysi I, Banfi C, Robin E, et al. ECMO as a bridge to decision: recovery, VAD, or heart transplantation? Int J Cardiol. 2015;187:620-7. 